\title{
WHITE DWARFS IN CATACLYSMIC BINARIES
}

\author{
Brian Warner \\ Department of Astronomy \\ University of Cape Town
}

\section{Introjuction}

For isolated stars, identification as a white dwarf may be effected in several ways. The fundamental property of abnormally low luminosity can be detected through direct measurement of trigonometric parallax or indirectly through large proper motion (accompanied by appropriate photometric properties). The presence of greatly pressure broadened absorption lines is another unambiguous criterion. Rapid light oscillations of the kind reviewed by Robinson are another hallmark of a select group of white dwarfs. Any or all of these criterja may be used to classify a star as a white dwarf and in general can be appiied to members of wide binary systems.

Interacting binaries, on the other hand, possess complications that prevent the diract application of these criterla; although all may be usable in various ways. In such systems the degenerate star is rot directly visible because (a) the companion may be the brighter by many magnitudes or (b) the result of the mass transference process outshines (or otherwise obscures) the degenerate component. In these circumstances the evidence for a white dwarf component is indirect. We revien this evidence here.

\section{Luminosities}

Estimates of the mean absolute magnitudes of cataclysmic variabies have been reviewed by the writer (Warner 1976b). Classical novae have $\overline{\mathrm{M}}_{v} \sim+4$ at minimum 1 ight and dwarf novae have $\bar{M}_{y} \sim+7.5$; in both classes there is a substantial spread about the mean. The combination of these low luminosities with the presence of very blue continua and enission lines led to the old spectral classifications of sdoe or sdBe for these objects. Such spectral types are now recognised to be inappropriate the spectra do not arise from stellar surfaces but from shock-heated or energy-dissipating viscous gas flows. However, the absolute magnitudes imply either lower main sequence components or degenerate stars. In those systems where a normal stellar absorption spectrum is present, the radial velocity variations show that the blue continuum and emission lines arise in the companion star. In general for novae, nova-like and dwarf novae (but not recurrent novae, in which the hotter star may be an accreting main sequence star - Webbink 1976) we deduce that the component carrying the high eneryy spectrum is of low luminosity and probably degenerate. 
Mass leterminations, which mostly make use of the assumption of Roche geometry, have been discussed by Ritter (1976), Robinson (1976a, 1976b) and Warner $(1973,1976 \mathrm{~b})$. Some further results have been obtained since these were published. The group of dwarf and classical novae with orbital periods greater than 3 hours for which reliable mass estimates have been made is listed in Table $I$. In a few cases the masses have been obtained from double-lined spectra, for which the assumption that the secondary fills its Roche lobe is either absent or required only for extraction of the orbital inclination. In the other cases, a massradius relationship for the secondary must be adopted, for which main sequence calculations are used. Although in some cases there may be evidence or suspicion for departure from the normal dwarf sequence (Warner 1978, Kiplinger 1979), there is in general good agreement between "direst" determinations and those assuming Roche geometry.

Additional support comes from observations of the secondary component in $U$ Gem. The Roche geometry approach gives a mean density $\bar{\rho}=6.4$ $\mathrm{gm} \mathrm{cm}^{-3}$ for the secondary of $U$ Gem (a result that depends only on the orbital period of the system: Faulkner et al. 1972) which indicates a spectral type of M3-4V (Allen 1973). Spectrophotometry of U Gem in the infrared (Wade 1979, Stauffer et al. 1979) shows the presence of an M4.5 dwarf.

In the case of the ultrashort period cataclysmic var'ailes (orbital period $₹ 2$ hours), all of which are dwarf novae, no reliable mass determinations are yet available. This is partly a result of the difficulty of measuring the small radial velocity amplitudes but also arises from confusion between the orbital velocity variations and the $S$-wave produced by the hot spot. Estimates based on measured $K_{1}$ values that do not properly allow for this latter confusion tend to give primary masses in excess of $1.4 M_{0}$ (Warner 1973; Smak 1979a). For WZ Sge, however, the spectroscopic evidence is less ambiguous and satisfactory masses should be obtainable; at present, however, there is disagreement on the precise interpretation of the observations (Robinson et al. 1978; Fabian et al. 1978; Ritter 1978; Ritter and Schröder 1979; Smak 1979b), but a primary mass below $1.4 M_{0}$ is permitted (Smak gives $M_{1}>0.31 M_{0}$ ) and possibly $\left.M_{1}>0.52 M_{0}\right)$.

In summary, for classical and dwarf novae the evidence at present provides no conflict with the $1.4 \mathrm{M}_{\Theta}$ theoretical upper 1 imit on mass for a white dwarf. Although spread over a range of a factor of two, the primary masses average $1.0 \mathrm{M}_{\odot}$ and much of the dispersion may be attributable to uncertainty in the reduction procedure. The primaries are considerably more massive than field white dwarfs, which average $0.65 M_{\odot}$ (Weidemann 1968).

Information for recurrent novae is sparse. Only for TCrB has a mass estimate been made; the hot star has a mass of $1.9 \mathrm{M}_{\odot}$ but the uncertainty is large because of the small radial velocity amplitude. As a result of this large mass, and based on his interpretation of the outburst light curve, Webbink $(1976,1977)$ concludes that TCrB contains a main sequence star rather than a white dwarf. 
Table 1

Masses of Classical (N) and Dwarf Novae (DN)

\begin{tabular}{|c|c|c|c|c|c|c|}
\hline Star & Type & Orbital & Period & $M_{2} / M_{0}$ & $M_{2} / M_{0}$ & Reference \\
\hline BV Cen & DN & $14^{\mathrm{h}}$ & $38^{6}$ & $1.6 \pm 0.2$ & $1.6 \pm 0.2$ & Vogt (1979c) \\
\hline RU Peg & DN & 8 & 54 & 1.12 & 0.97 & $\begin{array}{l}\text { Warner (1976b) } \\
\text { Kiplinger (1979) }\end{array}$ \\
\hline EM Cyg & $\mathrm{DN}$ & 7 & 00 & $0.90 \pm 0.17$ & $0.70 \pm 0.18$ & Robinson (1974) \\
\hline 2 Cam & $\mathrm{DN}$ & 6 & 56 & $0 . \dot{85}$ & 1.20 & Robinson (1973) \\
\hline SS Cyg & $\mathrm{DN}$ & 6 & 38 & 0.80 & 1.07 & Kiplinger (1979) \\
\hline RX And & DN & 5 & 05 & 0.65 & 1.02 & Warner $(1976 \mathrm{~b})$ \\
\hline DQ Her & $\mathrm{N}$ & 4 & 39 & 0.58 & 1.09 & Robinson (1976b) \\
\hline SS Aur & DN & 4 & 20 & 0.57 & 0.89 & Warner $(1976 \mathrm{~b})$ \\
\hline U Gem & DN & 4 & 10 & 0.35 & 0.9 & Smak $(1976)$ \\
\hline V603 Aq1 & N & 3 & 19 & 0.40 & 0.87 & Warner (1976b) \\
\hline
\end{tabular}

Line Profizes

Among the cataclysmic variables at minimum light, only the nova remnant DI Lac and the dwarf nova WZ Sge show broad shallow absorption lines, but these probably arise in the atmospheres of accretion discs (Warner 1976b). In many UX UMa stars, and in dwarf novae during outburst, broad shallow Balm ir absorptions are observed. The maximum widths of these lines sometimes e:zeeds $100 \AA$. A combination of pressure broadening and Doppler broadening in the inner regions of the disc, with a value of $M / R$ similar to that of a white dwarf is indicated (Warner (1976a).

Detailed ccmparison between Balmer absorption line profiles and these observations, using realistic model atmnspheres on accretion discs of various complexities (Koen 1976; Herter et al. 1979; Kiplinger 1978; Mayo et al. 1978 and this Conference), have shown encouraging agreement. When sufficiently realistic models are available (which must include an accurate description of the innermost region of the disc, where pressure and Doppler effects are maximised) it may well become possible to determine masses of the central stars and inclinations of their accretion discs.

Emission lines from the discs in cataclysmic variables have already been reviewed (Warner 1976b). Wings of the Balmer lines may extend out to $\sim 4500 \mathrm{~km} \mathrm{~s}^{-1}$, corresponding to the Keplerian velocity at the surface of a white dwarf with mass $\sim 1 \mathrm{M}_{0}$.

Eclipse Observations

The presence of eclipses in some of the cataclysmic variables raises the hope that direct measurement of the dimensions of the hot component may be made. In the first objects studied (e.g. U Gem: Warner and Nather 1971), the eclipses lacked the signature expected of a white dwarf:- viz. a rapid drop in brightness in less than a minute. This absence was one of the clues that led to the interpretation of the eclipse light curves as being principally of discs and not spots.

However, there are now two objects, both with ultrashort orbital periods and large inclinations, which clearly show the presence of an object with dimensions $\sim 10^{9} \mathrm{~cm}$. The first to be discussed was $Z$ Cha (Warner 
1974a), in which almost half of the ingress drop in brightness occurs in $\sim 40$ secs, and a similar recovery is seen some 345 secs later (see also Bailey 1979). The second object, recently discovered, is OY Car (Vogt 1979a) in which ingress and egress of the hot component take about 30 secs. In both of these systems, about half of the light at minimum comes from an object with dimensions $\sim 10^{9} \mathrm{~cm}$ (each also contains a hot spot with dimensions $\sim 10^{9} \mathrm{~cm}$ which is also seen to be separately eclipsed). In these stars, therefore, it appears possible to obtain direct information about the surface layers of the accreting star.

\section{Rapid Oscillations}

It is now we11-known that many cataclysmic variables, especially dwarf novae during outburst, show rapid oscillations in brightness (Warner 1979). A list of these stars is given in Table II. References for these may be found in previous reviews (Warner 1976b, 1979), to which should be added the following: SS Cyg (Cordova et al. 1979a), AH Her (Steining et al. 1979), WZ Sge (Robinson et al. 1978; Middleditch and Nelson 1978), VW Hyi (Robinson and Warner 1979), V533 Her (Patterson 1978), DQ Her (Patterson et al. 1978), U Gem (Cordova et al. 1979b), $A E$ Aqr, V Sge and AM CVn (Patterson 1979b).

From the dimensional relationship $t \sim(G \bar{D})^{-\frac{1}{2}}$ for gravitationally controlled processes, we deduce that the mean densities of the objects involved in produc:ing these oscillations 1 ie in the range $2 \times 10^{3}$ $2 \times 10^{5} \mathrm{gm} \mathrm{cm}^{-3}$, with the median at $\sim 2 \times 10^{4} \mathrm{gm} \mathrm{cm}^{-3}$. These values const+lute the most powerful evidence that the cataclysmic variables contain a degenerate object. When the precise mechanism of the oscillations can be identified, this argument may be turned around to give masses and/or radii of the white dwarfs.

It is important to note that the range of periods seen in cataclysmic variables lies entirely outside those observed in the $\mathrm{ZZ}$ Ceti stars. Although the white dwarfs in the CVs are of higher mass than the ZZ Cetis, the difference is certainly not sufficient to account for the different ranges of period. A different oscillation mechanism must be acting in the two classes of object. This conclusion is borne out by consideration of other aspects of the oscillations. With the exception of the nova remnants DQ Her and V533 Her, for whose periodic brightness variations a rotational origin may be required, the rates of change of periods show that the whole body of the white dwarf cannot be involved in the oscillation. Either the outer envelope of the white dwarf alone is excited (Warner and Brickhill 1978; Papaloizou and Pringle 1978) or the inner regions of the accretion disc and/or the transition region develop instabilities resulting in hot spots (Bath et al. 1974; Cordova et al. 1979a).

It is in this area that rapid oscillations will prove most valuable: the periods imply that the seat of the oscillations must be near or in the complicated zone between the accretion disc and the. white dwarf. A large fraction of the energy dissipated in the accretion disc emerges from this zone at temperatures $\sim 3 \times 10^{5} \mathrm{~K}$ (Pringle 1977). X-rays characteristic of this temperature have been observed in SS Cyg (Cordova et al. 1979a and this Conference) and were found to be periodically modulated by up to 100 percent. The dissipation of energy and the periodic oscillations are thus intimately related. Models of the transition zone 
Rapid Oscillations in Cataclysmic Variables

\begin{tabular}{lccc} 
Star & Type & $\frac{\text { Period range }}{\text { (secs) }}$ & Quasi Period \\
\cline { 2 - 3 } & & $8.8-9.7$ & $\sim 32$ \\
SS Cyg & DN & $11.6-11.9$ & $\sim 50$ \\
RU Peg & DN & $16.0-18.8$ & \\
Z Cam & DN & 16.6 & \\
EM Cyg & DN & $18.5-32.0$ & \\
CD -42014462 & NL & $20.0-20.5$ & \\
V436 Cen & DN & $20.3-33.8$ & \\
SY Cnc & DN & $22.5-29.5$ & $82-147$ \\
KT Per & DN & $24.1-31.5$ & \\
AH Her & DN & $24.3-33.0$ & \\
CN Ori & DN & $26.3,112.8-121.0$ & \\
AM CVn & NL & 27.0 & \\
YZ Cnc & DN & 27.7 & \\
2 Cha & DN & 27.87 .28 .98 & \\
WZ Sge & DN & $28-413$ \\
VW Hyi & DN & 33.1 & \\
AE Aqr & NL & 47.7 & \\
V Sge & NL & $63.6: 303$ & \\
V533 Her & N & 71.0659 & \\
DQ Her & N & & $29,70-150$ \\
U Gem & DN & &
\end{tabular}

(Durisen 1977; Durisen et al.: this Conference) are now required to reproduce the oscillatory instability. Intercomparison with the $X$-ray and optical observations may then lead to a refinement of the properties of white dwarfs and their accretion discs in CVs.

\section{$X-$ Ray Emission}

The deep gravitational potential well of the white dwarf, into which matter from the companion is falling, leads to the prediction of soft X-ray emission either from the hot spot (Warner 1974b) or from the inner disc (Pringle 1977). Soft X-rays have been detected from SS Cyg (Cordova et $a$ Z. 1979a and references therein) and U Gem (Cordova et al. $1979 \mathrm{~b}$ and references therein); the former is detected at minimum light and increases in emission during outburst; the latter is detectable only during outbursts. Detections of soft X-rays have also been made from the dwarf nova AY Lyr (Cordova and Garmire 1979) and the nova-like variable MV Lyr (Mason et al. 1979). Cordova et al. (1979c) have given upper limits on soft $X$-rays from twenty five dwarf novae during outburst.

In contrast, the detection of hard $X$-rays from cataclysmic variables was less well anticipated (although the higher energetics of the ultrashort period cataclysmics, which could lead to detectable hard X-rays, was pointed out by Warner (1972)). Hard X-rays have been detected at minimum light from the dwarf novae Ex Hya (Watson et al. 1978, Bradt et al. 1979), U Gem (Swank et al. 1978) and SS Cyg (Swank et al. 1978, and references therein); from the nova-like variable identified with 2A0526-328 (Charles et al. 1979), and from GK Per during minor outburst 
(King et al. 1979). Watson et al. (1978) give upper limits on the hard $\mathrm{X}$-ray emission from 45 other dwarf novae.

Two mechanisms have been proposed for the emission of hard X-rays from a white dwarf. Ricketts et al. (1978) propose that the white dwarf must have a magnctic field $\sim 10^{6}$ gauss which controls the accretion flow in the neighbourhood of the white dwarf, thus producing radial infall from which hard X-rays can be generated (Fabian et al. 1976). Pringle and Savonije (1979), on the other hand, find that hard X-rays may be produced from a normal transition zone between accretion disc and white dwarf if the decelerating shock waves are strong and the accretion rate is not too high.

The availability of X-rays over a wide range of energies, and their behaviour during outbursts of dwarf novae (and of the star GK Per, which is both a nova remnant and a dwarf nova) opens a new window for the study of white dwarfs undergoing accretion.

\section{Oxbital Periods}

In listing the cataclysmic variables with known orbital periods in 1975 it was pointed out (Warner 1976b) that there was a significant absence of periods between $2 \mathrm{~h}$ and $3 \mathrm{~h}$. The number of known periods has increasiu by fifty percent since that list was prepared. Although the gap has been narroweil slightly, there is still a highly significant deficiency of periods in the 2-3 hour range (Table III - this omits TCrB for redions given earlier. References are given only if additional to the author's earlier review.)

Webbink (1979) has interpreted the gap as arising from a discontinuity in core masses for the progenitor stars and concludes that the ultrashort period systems $(\mathrm{P} \approx 2 \mathrm{~h})$ must contain helium white dwarfs, whereas the longer period $\left(P>3^{h}\right)$ systems contain carbon-oxygen white dwarfs.

In view of this suggested dichotomy of composition, it is important to note the correlation between orbital period and outburst properties evident in Table III. Whereas in 1975 there were no apparent correlations (except for the hint of a preference of SU UMa stars for ultrashort periods - Warner 1975), the addition of more stars and reclassification of some (notably WZ Sge, moved from the recurrent nova class to the dwarf nova, probable subtype SU UMa: Warner 1976b, Patterson 1979a) has resulted in several distinct interrelations. Namely, (i) all novae and UX UMa stars have $P>3 \mathrm{~h}$, (ii) among the dwarf novae those with $P>3^{h}$ are all normal or $Z$ Cam stars whereas those with $\mathrm{P} ₹ 2^{\mathrm{h}}$ are all SU UMa stars, (iii) the AM Her stars are the only ones that bridge the gap.

Whether there is a causal relationship between these properties and Webbink's proposed dichotomy of white dwarf structure is not evident. It is equally possible that the SU UMa type outbursts that occur in the ultrashort period group are a result of the lower mass secondaries, the different mass ratios, or the closer separations. The SU UMa phenomenon (Warner 1975) is an addition to normal dwarf nova behaviour; the latter is not affected by the peculiar circumstances occurring in the ultrashort systems. 
Table III

Orbital Periods of Cataclysmic Variables

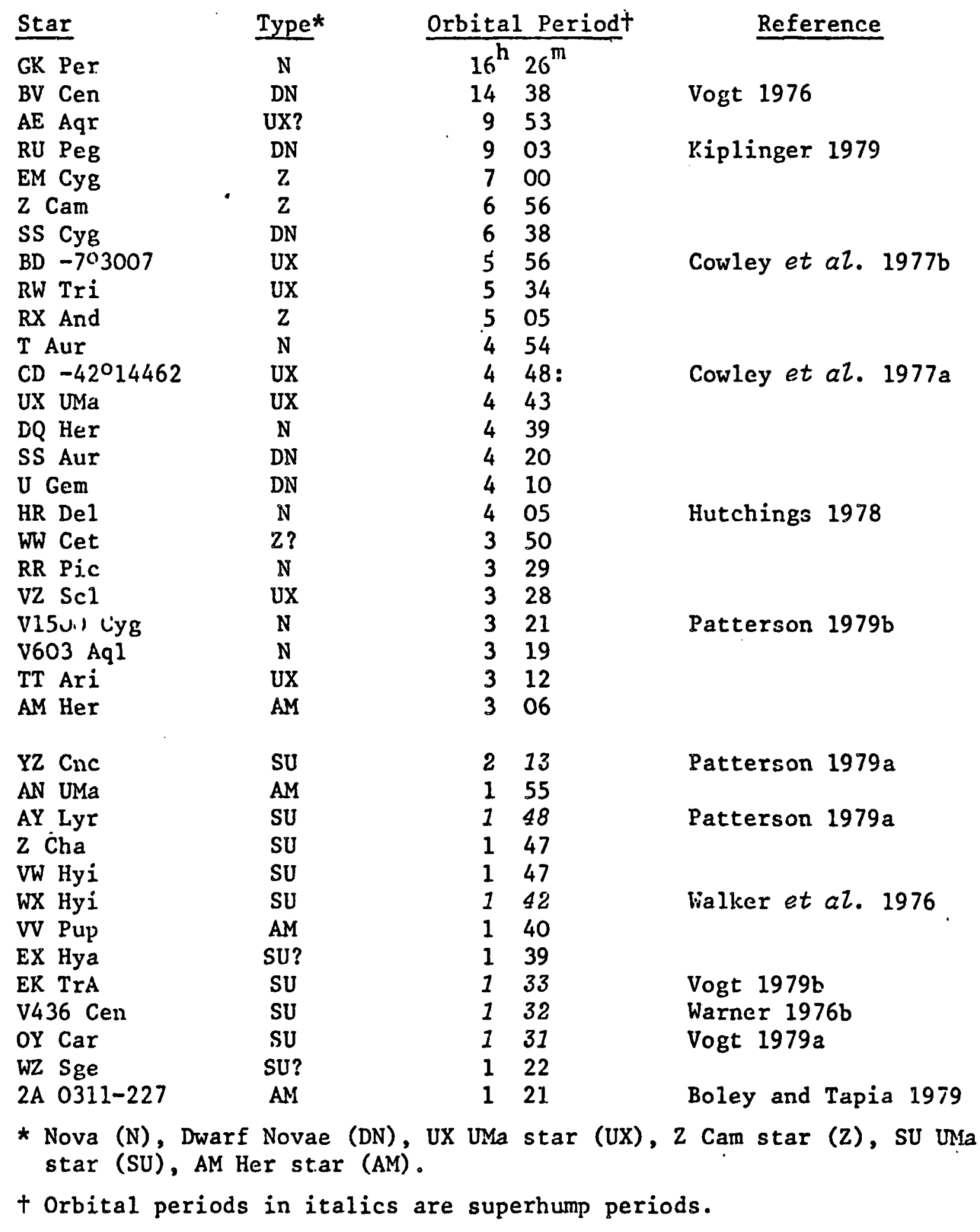

\section{REFERENCES}

Allen, C.W. 1973, Astrophysical Quantities 3rd Edjtion, Athlone Press, London.

Bailey, J. 1979, Mon. Not, R, astr. Soc., 187, 645.

Bath, G.T., Fvans, W.D. and Papaloizou, J. 1974, Ion. Not. R. astr. Soc., $167,7 P$. 
Boley, F. and Tapia, S. 1979, I.A.U. Circ. No. 3327

Bradt, H.V., Doxsey, R.E. and Jernigan, J.G., 1979, Adv. Sp. Exp1. 3, in press.

Charles, P., Thorstensen, J., Bowyer, S. and Middleditch, J. 1979, Astrophys. J. Letts. In press.

Cordova, F. and Garmire, G. 1979, preprint.

Cordova, F., Chester, T.J., Tuohy, I.R. and Garmire, G.P. 1979a, preprint.

Cordova, F., Chester, T., Tuohy, I., Garmire, G. and Mason, K. 1979b, Bull. Amer. Astr. Soc., 11, 393.

Cordova, F.A., Nugent, J.J., Klein, S.R. and Garmire, G.P. 1979c, Mon. Not. R. astr. Soc. In press.

Cowley, A.P., Crampton, D. and Hesser, J.E. 1977a, Astrophys. J., 214, 471 .

Cowley, A.P., Crampton, D. and Hesser, J.E. 1977b, Pub1. Astr. Soc. Pacific, 89, 716.

Durisen, R. H. 1977, Astrophys. J., 213, 14.5 .

Fabian, A.C., Pringle, J.E. and Rees, M.J. 1976, Mon. Not. R. astr. Soc., $175,43$.

Fabian, A.C., Lin, D.N.C., Papaloizou, J., Pringle, J.E. and Whelan, J.A.J. 1978 , Mon. Not. R. astr. Soc., 184, 835.

Faulkner, J., Flannery, B.P. and Warner, B. 1972, Astrophys. J. Letts., 175, L79.

Herter, T., Lacasse, M.G., Wesemael, F. and Wirget, D.E. 1979, Astrophys. J. Supp. Series, $39,513$.

Hutchings, J.B. $19 \overline{79}$, I.A.U. Circ. No. 3310.

King, A.R., Ricketts, M.J. and Warwick, R.S. 1979, Mon. Not. R. astr. Soc., 187, 77P.

Kiplinger, A.L. 1978, Ph.D. Thesis, University of Texas.

Kiplinger, A.L. 1979 , Astron. J., 84, 655.

Koen, M.C. 1976, M.Sc. Dissertation, University of Cape Town.

Mason, K.O., Kahn, S.M. and Bowyer, C.S. 1979, Nature, in press.

Mayo, S.K., Wickramasinghe, D.T. and Whelan, J.A.J. 1978, Symp. No. 46, Int. Astr. Union, Hamilton, New Zealand.

Middleditch, J. and Nelson, J. 1978, Bull. Am. Astr. Soc., 10, 632 .

Papaloizou, J. and Pringle, J.E. 1978, Mon. Not. R. astr. Soc., 182, 423.

Patterson, J. 1978, I.A.U. Circ. No. 3283.

Patterson, J. 1979a, Astron. J., in press.

Patterson, J. 1979b, Bull. Amer. Astr. Soc., 11, 335.

Patterson, J., Robinson, E.L. and Nather, R.E. 1978, Astrophys. J., 224, 570.

Pringle, J.E. 1977, Mon. Not. R. astr. Soc., 178, 195.

Pringle, J.E. and Savonije, G.J. 1979, Mon. Not. R. astr. Soc., 187, 777.

Ricketts, M.J., King, A.R, and Raine, D.J. 1978, Mon. Not. R. astr. Soc. in press.

Ritter, H. 1976, Mon. Not. R. astr. Soc., 175, 279.

Ritter, H. 1978, Astr. Astrophys., 68, 455.

Ritter, H. and Schröder, R. 1979, Astr. Astrophys., in press.

Robinson, E.I. 1973, Astrophys. J., 180, 121.

Robinson, E.L. 1974, Astrophys. J., $\overline{19} \overline{3}, 191$.

Robinson, E.L. 1976a, Adv. Astr. Astrophys., 14, 119.

Robinson, E.L. 1976b, Astrophys. J., 203, 485 .

Robinson, E.L. and Warner, B. 1979, in preparation.

Robinson, E.L., Nather, R.E. and Patterson, J. 1978, Astrophys. J., 219, 168. 
Smak, J. 1976, Acta. Astr., 26, 277.

Smak, J. 1979a, preprint.

Smak, J. 1979b, Acta Astr., 29, in press.

Stauffer, J., Spinrad, H. and Thorstensen, J. 1979, Publ. Astr. Soc. Pacific, $91,59$.

Steining, R.F., Hildebrand, R.H. and Spillar, E.J. 1979, preprint.

Swank, J.H., Boldt, E.A., Holt, S.S., Rothschild, R.E. and Serlemitsos, P.J. 1978, Astrophys. J., 226, 377.

Vogt, N. 1976, Symp. No. 73, Int. Astr. Union, Cambridge, p. 147.

Vogt, N. 1979a, The Messenger, No. 17, p. 39 and I.A.U. Circ. No. 3357. Vogt, N. 1979b, I.A.U. Circ. No. 3375 .

Vogt, N. 1979c, In I.A.U. Colloquium No. 46, Hamilton, New Zealand, in press.

Wade, R.A. 1979, Astron. J., 84, 562.

Walker, W.S.G., Marino, B.F. and Freeth, G. 1976, Inf. Bull. Var. Stars No. 1185 .

Warner, B. 1972, Mon. Not. R, astr. Soc., 158, 425.

Warner, B. 1973, Non. Not. R. astr. Soc., $\overline{162}, 189$.

Warner, B. 1974a, Mon. Not. R. astr. Soc., $\overline{168}, 235$.

Warner, B. 1974b, Mon. Not. R. astr. Soc., $\overline{167}, 47 \mathrm{P}$ 。

Warner, B. 1975, In I.A.U. Colloquium No. 29, Budapest, p. 247.

Warner, B. 1976a, Observatory, 96, 49.

Warner, B. 1976b, Int. Astr. Un. Symp. No. 73, Cambridge, England, p.85. Warner, B. 1978, icta Astr., 28, 303.

Warner, B. 1979, Symp. No. 46, Int. Astr. Union, Hamilton, New Zealand, isc pless.

Warner, B. and Brickhill, A.J. 1978, Mon. Not. R. astr. Soc., 182, 777.

Warner, B. and Nather, R.E. 1971, Mon. Not. R. astr. Soc., 152, 219.

Watson, M.G., Snerrington, M.R. and Jameson, R.F. 1978, Mon. Not. R. astr. Soc., 184, 79P.

Webbink, R.F. 1976, Nature, 262, 271.

Webbink, R.F. 1977, Publ. Var. Star Section, R.A.S. of New Zealand, No. 5, p. 22 .

Webbink, R.F. 1979, In I.A.U. Colloq. No. 46, Hamilton, New Zealand, in press.

Weidemann, V. 1968, Ann. Rev. Astr. Astrophys., 6, 351.

Wiliiams, R.E. 1979, preprint. 